\title{
The effect of distant large scale structure on weak lensing mass estimates
}

\author{
H. Hoekstra* \\ Kapteyn Astronomical Institute, University of Groningen, PO Box 800, 9700 AV Groningen, The Netherlands
}

Received 27 July 2000 / Accepted 19 February 2001

\begin{abstract}
We quantify the uncertainty in weak lensing mass estimates of clusters of galaxies, caused by distant (uncorrelated) large scale structure along the line of sight. We find that the effect is fairly small for deep observations $(20<R<26)$ of massive clusters $\left(\sigma=1000 \mathrm{~km} \mathrm{~s}^{-1}\right)$ at intermediate redshifts, where the bulk of the sources are at high redshifts compared to the cluster redshift. If the lensing signal is measured out to $1.5 h_{50}^{-1} \mathrm{Mpc}$ the typical $1 \sigma$ relative uncertainty in the mass is about $6 \%$. However, in other situations the induced uncertainty can be larger. For instance, in the case of nearby clusters, such as the Coma cluster, background structures introduce a considerable uncertainty in the mass, limiting the maximum achievable signal-to-noise ratio to $\sim 7$, even for deep observations. The noise in the cluster mass estimate caused by the large scale structure increases with increasing aperture size, which will also complicate attempts to constrain cluster mass profiles at large distances from the cluster centre. However, the distant large scale structure studied here can be considered an additional (statistical) source of error, and by averaging the results of several clusters the noise is decreased.
\end{abstract}

Key words. cosmology - gravitational lensing - galaxies: clusters: - dark matter

\section{Introduction}

Light rays are deflected as they travel through the universe, and as a result our view of the distant, faint galaxies is somewhat distorted. Measuring these lensing induced distortions has proven to be a valuable tool in observational cosmology (see the review by Mellier 1999): the lensing signal is sensitive to the projected mass surface density of the deflector, and through lensing one can probe the mass distribution without having to rely on assumptions about the dynamical state and nature of the deflecting matter.

When the surface density of the lens is sufficiently high, the distortions can be so large that multiple images can be seen, which provide strong constraints on the mass distribution in cluster cores. In this paper we will concentrate on the weak lensing regime, which allows us to study the cluster mass distribution on larger scales.

Weak lensing is a powerful tool to probe the distribution of matter in the universe on various scales. One exciting application is the measurement of the lensing signal induced by large scale structure (e.g. Blandford

\footnotetext{
Send offprint requests to: H. Hoekstra, e-mail: hoekstra@astro.rug.nl

* Present address: CITA, University of Toronto, 60 St. George Street, M5S 3H8 Ontario, Canada
}

et al. 1991; Kaiser 1992; Bernardeau et al. 1997; Schneider et al. 1998, SWJK hereafter), and recently several groups have reported detections of this signal (Bacon et al. 2000; Kaiser et al. 2000; van Waerbeke et al. 2000; Wittman et al. 2000).

The first successful weak lensing results were obtained by studying the systematic distortion of the images of faint galaxies behind rich clusters of galaxies (e.g. Tyson et al. 1990). Measuring the masses and mass-to-light ratios of rich clusters is an important application of weak lensing, because comparison of the weak lensing result with other mass estimators (e.g., X-ray, S-Z effect, or velocities) can give important clues about the geometry and dynamical state of the system (e.g., Zaroubi et al. 1998).

Weak lensing also has some drawbacks. First of all, the amplitude of the lensing signal depends on the redshift distribution of the faint background galaxies. These galaxies are generally too faint to be included in spectroscopic redshift surveys, but photometric redshifts are thought to provide ample information on the redshift distribution of these galaxies (e.g., Hoekstra et al. 2000). Also the observed weak lensing signal is a measure of a surface density contrast, and one of the problems associated with this is the mass sheet degeneracy (Gorenstein et al. 1988). One could try to get around this problem by measuring 
the lensing signal out to large distances from the centre, and arguing that the cluster surface density should vanish there.

Another potential problem is the fact that the lensing signal is sensitive to all matter along the line of sight. In general, weak lensing analyses of clusters assume that the signal induced by the cluster under investigation is so dominant that the contribution from other structures along the line of sight can be neglected.

The effect of structures, close to the cluster, such as the filaments that connect the cluster to the "cosmic web", or an elongation along the line of sight, have been studied through N-body simulations (e.g., Cen 1997; Metzler et al. 1999). Local structures introduce both a bias and an additional uncertainty in the cluster mass estimate, in particular when one for instance tries to measure the mass within $r_{200}$.

In this paper we study how distant structures affect weak lensing mass estimates of clusters of galaxies. Unlike the local structure, these are uncorrelated with the cluster, and they can be filaments, galaxy groups, or even other clusters. That such structures affect the mass estimates is easily seen in the case of a massive high redshift cluster, with a low redshift group in front of it. Assume the cluster is at a redshift of unity, with a group at say $z=0.3$, and that the mass of the group is one tenth of that of the cluster: the contribution of the group to the total lensing signal is about one third, compared to the $10 \%$ in mass.

In this paper we will quantify the uncertainty in weak lensing mass estimates of rich clusters caused by intervening matter. To do so we examine the predicted statistical properties of the contribution by large scale structure. We use an approach similar to what is done to predict the amplitude of the cosmic shear signal, which is used to constrain the cosmological parameters. Given a cosmological model, we estimate the uncertainty in cluster masses derived from weak lensing analyses.

The structure of this paper is as follows. In Sect. 2 we discuss two popular methods to measure cluster masses. In Sect. 3 we derive the equations that describe the effect of large scale structure on such mass estimates. The effect of large scale structure on mass estimates for clusters is quantified in Sect. 4. We examine the dependence on cluster redshift, aperture size and source redshift distribution.

\section{Estimating masses from weak lensing}

Cluster masses can be measured in several ways. In this paper we will use aperture mass statistics, which provide a filtered measurement of the surface mass density in an aperture (Schneider 1996, S96 hereafter):

$M_{\mathrm{ap}}(\theta)=\int_{0}^{\theta} \mathrm{d}^{2} \theta^{\prime} U\left(\left|\theta^{\prime}\right|\right) \kappa\left(\theta^{\prime}\right)$,

where $U(\theta)$ is the weight or filter function. Provided that $U(\theta)$ is compensated, i.e.

$\int_{0}^{\theta} \mathrm{d} b b U(b)=0$, the aperture statistic can be related to the tangential shear $\gamma_{\mathrm{T}}$. Unfortunately $\gamma_{\mathrm{T}}$ cannot be observed directly, but one can measure the distortion $g_{\mathrm{T}}=\gamma_{\mathrm{T}} /(1-\kappa)$. In the weak lensing regime, where $\kappa \approx 0$, one can use $\gamma_{\mathrm{T}} \approx g_{\mathrm{T}}$, which is what we do throughout this paper. Then we have

$M_{\mathrm{ap}}(\theta)=\int_{0}^{\theta} \mathrm{d}^{2} \theta Q(|\theta|) \gamma_{\mathrm{T}}(\theta)$.

Here, $Q(\theta)$ is related to $U(\theta)$ through:

$Q(b)=\frac{2}{b^{2}} \int_{0}^{b} \mathrm{~d} b^{\prime} b^{\prime} U\left(b^{\prime}\right)-U(b)$.

S96 has shown that the signal-to-noise ratio of the aperture mass measurement is optimal when $Q(|\theta|)$ is the same as the (expected) tangential shear as a function of $|\theta|$.

We want to quantify the uncertainty in mass measurements of clusters, and therefore require that the aperture statistic has a clear physical meaning. Studies of cosmic shear (see SWJK) use different choices for $Q(|\theta|)$. Below we discuss two statistics that have been used to estimate cluster masses.

\subsection{SIS model fit}

A popular model to describe the cluster mass distribution is the singular isothermal sphere (SIS)

$\kappa(r)=\frac{r_{\mathrm{E}}}{2 r}$,

where $r_{\mathrm{E}}$ is the Einstein radius. The Einstein radius (in radians) is related to the velocity dispersion through

$r_{\mathrm{E}}=4 \pi\left(\frac{\sigma}{c}\right)^{2} \beta$.

The tangential shear as a function of radius is simply given by

$\gamma_{\mathrm{T}}(r)=\kappa(r)=\frac{r_{\mathrm{E}}}{2 r}$.

The best fit SIS model is obtained by minimizing

$\sum_{i}\left(\frac{\gamma_{\mathrm{T}, i}-r_{\mathrm{E}} /\left(2 r_{i}\right)}{\sigma_{\gamma}}\right)^{2}$

with respect to $r_{\mathrm{E}}$, where $\gamma_{\mathrm{T}, i}$ are the individual measurements of the lensing signal at radius $r_{i}$. The uncertainty in the measurement of the tangential shear, $\sigma_{\gamma}$ is given by

$\sigma_{\gamma}^{2}=\frac{\sigma_{\text {gal }}^{2}}{2 \pi \bar{n} r_{i} \Delta r_{i}}$

with $\sigma_{\text {gal }}$ the uncertainty in the measurement of an individual galaxy, $\bar{n}$ the number density of sources, and $\Delta r_{i}$ the width of the $i$ th radial bin. If we take the widths of all the bins the same, and assume that the number density of sources is uniform, minimizing with respect to $r_{\mathrm{E}}$ gives

$r_{\mathrm{E}}=2 \frac{\sum_{i} \gamma_{\mathrm{T}, i}}{\sum_{i} \frac{1}{r_{i}}}$ 
For a continuous profile the summation can be replaced by an integral. As mentioned before one measures the distortion and not the shear, and Eq. (10) only works well when $\kappa$ is small. Also substructure in the cluster core changes the average tangential distortion (e.g., Hoekstra et al. 2000). To come around these problems one has to choose an inner radius for the fit, such that Eq. (10) is a good approximation.

If we assume that the tangential shear has been measured from a distance $R_{0}$ out to $R$, the Einstein radius satisfies

$r_{\mathrm{E}}=\frac{2}{\ln \left(R / R_{0}\right)} \int_{R_{0}}^{R} \mathrm{~d} r \gamma_{\mathrm{T}}(r)$

Comparison with Eq. (3) shows that the best fit SIS model is an aperture mass measurement where $Q(|\theta|)$ is given by

$Q(|\theta|)=\left\{\begin{array}{cl}\left(\pi \theta \ln \left(R / R_{0}\right)\right)^{-1}, & R_{0} \leq|\theta| \leq R \\ 0 \quad, & \text { elsewhere }\end{array}\right.$

The corresponding weight function for the surface density $U(\theta)$ is given by

$U(|\theta|)=\frac{1}{\pi \ln \left(R / R_{0}\right)}\left\{\begin{array}{cl}2 / R_{0}-2 / R, & 0 \leq|\theta|<R_{0} \\ 1 /|\theta|-2 / R, & R_{0} \leq|\theta| \leq R \\ 0 \quad, & |\theta|>R\end{array}\right.$

The mass profiles of many clusters that have been studied to date are consistent with a SIS model. Thus the weight function suggested above is close to the optimal choice. However, this choice for $U(|\theta|)$ is not a continuous function. A functional form which is continuous, yet similar is suggested by S96, who discusses the use of aperture mass statistics to find mass concentrations in deep images.

\subsection{S-statistic}

Another statistic which has been widely used is the $\zeta$ statistic (Fahlman et al. 1994):

$\zeta\left(\theta_{1}, \theta_{2}\right)=\bar{\kappa}\left(<\theta_{1}\right)-\bar{\kappa}\left(\theta_{1}<|\theta|<\theta_{2}\right)$.

It gives the difference of the average dimensionless surface mass density in a circular aperture of radius $\theta_{1}$ and that in an annulus with $\theta_{1}<|\theta|<\theta_{2}$. The corresponding weight function $U(|\theta|)$ is given by:

$U(|\theta|)=\left\{\begin{array}{cl}\left(\pi \theta_{1}^{2}\right)^{-1}, & |\theta|<\theta_{1} \\ -\left(\pi\left(\theta_{2}^{2}-\theta_{1}^{2}\right)\right)^{-1}, & \theta_{1}<|\theta|<\theta_{2} \\ 0 \quad, & \text { elsewhere }\end{array}\right.$.

This gives

$Q(|\theta|)=\left\{\begin{array}{cl}\theta_{2}^{2}\left(\pi \theta^{2}\left(\theta_{2}^{2}-\theta_{1}^{2}\right)\right)^{-1}, & \theta_{1}<|\theta|<\theta_{2} \\ 0 \quad, & \text { elsewhere }\end{array}\right.$.

Thus the $\zeta$-statistic can be related to the tangential shear through:

$\zeta\left(\theta_{1}, \theta_{2}\right)=\frac{2 \theta_{2}^{2}}{\theta_{2}^{2}-\theta_{1}^{2}} \int_{\theta_{1}}^{\theta_{2}} \mathrm{~d} \ln \theta\left\langle\gamma_{\mathrm{T}}\right\rangle(|\theta|)$.
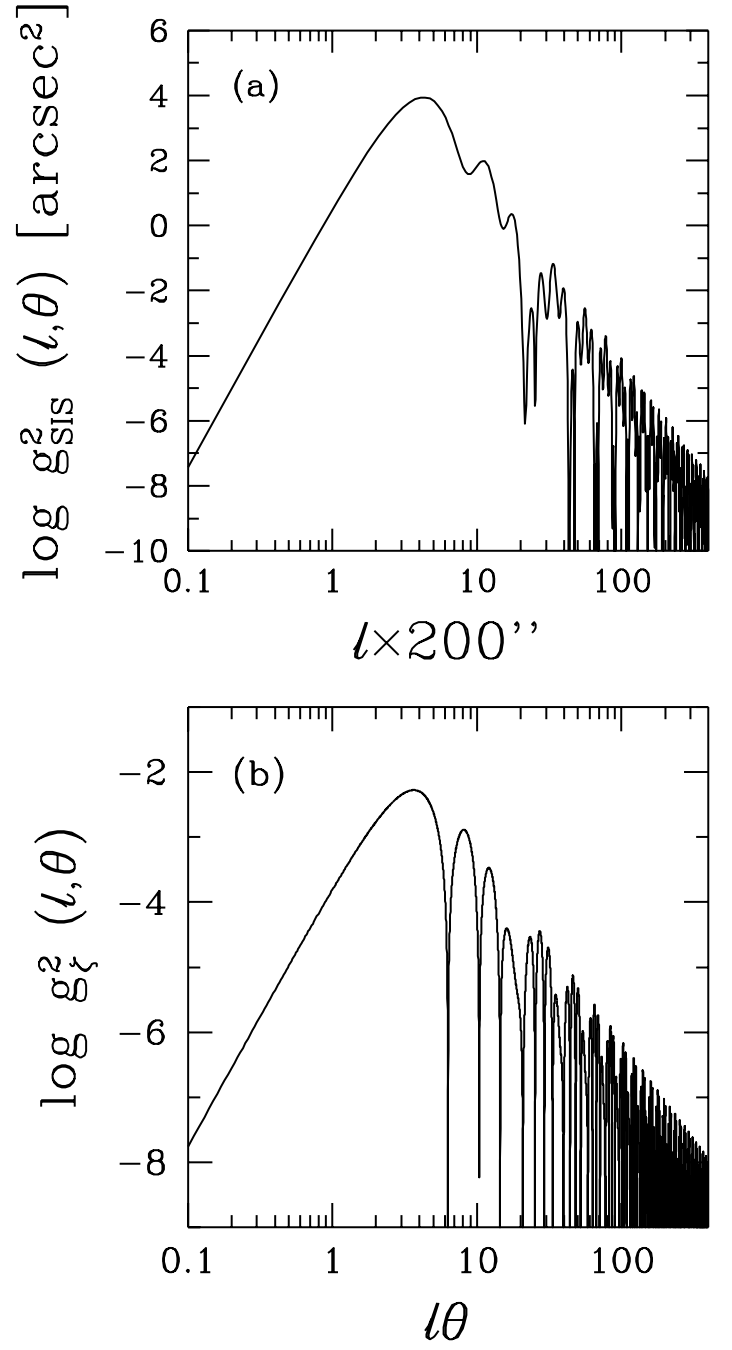

Fig. 1. a) The function $g_{\mathrm{SIS}}^{2}(l, \theta)$ as a function of $l \theta$ for $\alpha=$ 0.15 , and $\left.\theta=200^{\prime \prime}, \mathbf{b}\right) g_{\zeta}^{2}(l, \theta)$ for $\alpha=\frac{2}{3}$. Both curves show similar behaviour, with $g_{\zeta}^{2}(l, \theta)$ peaking at a similar values of $l \theta$

The SIS model fit has only a simple physical meaning when the cluster mass profile is indeed isothermal. The interpretation of the $\zeta$-statistic is independent of the mass distribution, although the statistic is not optimal in terms of signal-to-noise ratio.

Under the assumption that one can estimate or minimize $\bar{\kappa}\left(\theta_{1}<|\theta|<\theta_{2}\right)$, the average surface density in the control annulus, one obtains a measurement of $\bar{\kappa}\left(<\theta_{1}\right)$, and thus of the cluster mass. One way to do so is to assume a model for the cluster surface mass density which is used to estimate the average surface density in the annulus (e.g. Hoekstra et al. 1998). This way the cluster mass estimate is less dependent on the assumed model compared to the SIS model fit.

\section{Contribution by LSS}

Under the assumption that the cluster is the only lensing structure in the field, the aperture mass $M_{\mathrm{obs}}$ provides 
a direct measure of the cluster mass. However, the weak lensing signal is sensitive to all matter along the line of sight, and other structures, which we will refer to as large scale structure, will introduce an additional signal:

$M_{\mathrm{obs}}(\theta)=M_{\text {cluster }}(\theta)+M_{\mathrm{LSS}}(\theta)$.

The aperture mass statistic measures a density contrast, and the expectation value for the contribution by uncorrelated large scale structure $\left\langle M_{\mathrm{LSS}}\right\rangle=0$ (see also SWJK). Thus on average, distant structures do not introduce a bias in cluster mass estimates, but an uncertainty in the cluster mass of $\left\langle M_{\mathrm{LSS}}^{2}\right\rangle^{1 / 2}$. As a result the effect of distant large scale structure can be treated as an additional (statistical) source of error. Its relative importance can be decreased by averaging the results for several clusters, i.e. measuring their average mass. We note that on certain scales $M_{\text {LSS }}$ might be distributed skewly.

The effect of "local" (or correlated) large scale structure has been studied using $N$-body simulations (e.g., Cen 1997; Metzler et al. 1999). In this case either projection effects, or filaments connected to the cluster bias the weak lensing mass estimates towards somewhat higher values. This becomes particularly important when one tries to estimate the enclosed mass within several Mpc's from the cluster centre (Cen 1997).

SWJK have shown how one can relate the aperture mass statistic to the power spectrum of density fluctuations. Measuring $\left\langle M_{\mathrm{LSS}}^{2}\right\rangle^{1 / 2}$ for a number of random fields allows one to constrain the projected power spectrum. In this paper the problem is reversed: given the power spectrum, what is the resulting $\left\langle M_{\mathrm{LSS}}^{2}\right\rangle^{1 / 2}$ ? A detailed discussion about the use of aperture mass statistics and cosmic shear can be found in SWJK and Bartelmann \& Schneider (1999).

SWJK showed that the effect of large scale structure on the variance in the aperture mass statistic is given by:

$\left\langle M_{\mathrm{LSS}}^{2}\right\rangle(\theta)=2 \pi \int_{0}^{\infty} \mathrm{d} l l P_{\kappa}(l) g(l, \theta)^{2}$,

where the effective projected power spectrum $P_{\kappa}(l)$ is given by:

$P_{\kappa}(l)=\frac{9 H_{0}^{4} \Omega_{m}^{2}}{4 c^{4}} \int_{0}^{w_{\mathrm{H}}} \mathrm{d} w\left(\frac{\bar{W}(w)}{a(w)}\right)^{2} P_{\delta}\left(\frac{l}{f_{K}(w)} ; w\right)$

Here $w$ is the radial coordinate, $a(w)$ the cosmic scale factor, and $f_{\mathrm{K}}(w)$ the comoving angular diameter distance. $\bar{W}(w)$ is the source averaged ratio of angular diameter distances $D_{l s} / D_{s}$ for a redshift distribution of sources $p_{w}(w)$ :

$\bar{W}(w)=\int_{w}^{w_{\mathrm{H}}} \mathrm{d} w^{\prime} p_{w}\left(w^{\prime}\right) \frac{f_{\mathrm{K}}\left(w^{\prime}-w\right)}{f_{\mathrm{K}}\left(w^{\prime}\right)}$.

The function $g(l, \theta)$ in Eq. (19) depends on the filter function $U(|\theta|)$, and is given by:

$g(l, \theta)=\int_{0}^{\theta} \mathrm{d} \phi \phi U(\phi) J_{0}(l \phi)$
As has been demonstrated in Jain \& Seljak (1997) and SWJK it is crucial to use the non-linear power spectrum in Eq. (20). This power spectrum can be derived from the linearly evolved cosmological power spectrum following the prescriptions of Hamilton et al. (1991), and Peacock \& Dodds (1996).

\subsection{Filter functions}

The form of the function $g^{2}(l, \theta)$, which probes the projected power spectrum, depends on the choice of $U(|\theta|)$. For the SIS model fit we take $R_{0}=\alpha R$, which yields

$$
\begin{aligned}
g_{\mathrm{SIS}}(l, \theta)= & \frac{-\theta}{\pi \ln (\alpha) l \theta} \\
& \times\left[2\left\{J_{1}(\alpha l \theta)-J_{1}(l \theta)\right\}+\int_{\alpha l \theta}^{l \theta} \mathrm{d} s J_{0}(s)\right] .
\end{aligned}
$$

We will use a typical value for $\alpha=0.15$ (e.g., Hoekstra et al. 1998). Larger values are used when much substructure is found in the cluster core (e.g., Hoekstra et al. 2000). The resulting $g_{\text {SIS }}^{2}(l, \theta)$ is presented in Fig. 1a.

For the $\zeta$-statistic we find

$g_{\zeta}(l, \theta)=\frac{J_{1}(\alpha l \theta)-\alpha J_{1}(l \theta)}{\pi \alpha l \theta\left(1-\alpha^{2}\right)}$

where we have taken the inner radius $\theta_{1}=\alpha \theta$. A typical value for $\alpha$ used in weak lensing mass estimates of clusters is $\frac{2}{3}$, i.e., the control annulus runs from the boundary of the region in which we want to determine the mass out to 1.5 times this radius. Throughout this paper we will use this value for $\alpha$. In Fig. $1 \mathrm{~b}$ the filter function $g_{\zeta}^{2}(l, \theta)$ is shown (taking $\alpha=\frac{2}{3}$ ).

\subsection{Photometric redshifts}

The projected power spectrum $P_{\kappa}(k)$ depends on the redshift distribution of the sources, and consequently we need to know, or assume, a redshift distribution for the sources. We will use the photometric redshift distributions inferred from the northern and southern Hubble Deep Fields (Fernández-Soto et al. 1999; Chen et al. 1998), which generally work well (e.g., Hoekstra et al. 2000). There is, however, some field to field variation in the redshift distribution the sources, but we assume that the distributions obtained from the HDFs are representative for the whole sky. We note that most of the lensing by large scale structure is caused by structures at intermediate redshifts, and as a result the signal induced by the LSS is fairly robust against variations in the source redshift distribution.

Using the colours of the galaxies in the HDFs we have computed their $R$ magnitudes, which allows a direct comparison with ground based observations. We use galaxies fainter than $R=20$. The resulting photometric redshift distributions for different limiting magnitudes are presented in Fig. 2. 


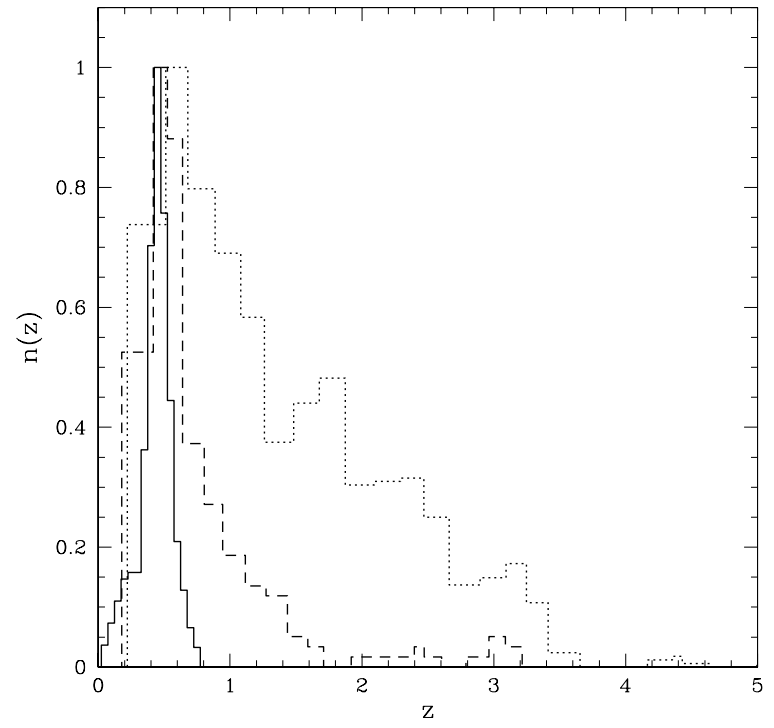

Fig. 2. Photometric redshift distributions from the HDF north and south. The counts have been normalized to a peak value of unity. The solid line is for galaxies with $20<R<22$, the dashed line is for $20<R<24$, and the dotted line for $20<R<26$. These distributions are used to compute the lensing signals and the contribution of large scale structure

\subsection{Aperture statistics}

Given the photometric redshift distribution discussed above we calculate the contribution of the LSS to the measurements of $r_{\mathrm{E}}$ and the $\zeta$-statistic as a function of aperture size. We have considered three different cosmologies, which are listed in Table 1.

Table 1. Parameters of the cosmological models considered here. All three models are cluster normalized

\begin{tabular}{ccccc}
\hline \hline & $h$ & $\Omega_{\mathrm{m}}$ & $\Omega_{\Lambda}$ & $\sigma_{8}$ \\
SCDM & 0.5 & 1.0 & 0.0 & 0.5 \\
OCDM & 0.7 & 0.3 & 0.0 & 0.85 \\
$\Lambda \mathrm{CDM}$ & 0.7 & 0.3 & 0.7 & 0.90 \\
\hline \hline
\end{tabular}

Figure 3a shows the scatter in the value of the Einstein radius $r_{\mathrm{E}}$ as a function of aperture size for the SCDM (thick lines) and the OCDM (thin lines) models. For clarity, we limit the number of curves shown in Fig. 3 and omit the results for the $\Lambda \mathrm{CDM}$ model. For this model the scatter is similar to the OCDM model, with a somewhat higher amplitude on large scales and a somewhat lower amplitude on arcminute scales. The scatter in the value of $r_{\mathrm{E}}$ increases for larger aperture sizes. The value of $r_{\mathrm{E}}$ is independent of aperture size, and as a result the relative contribution of large scale structure to the error budget increases with increasing aperture size. Also the scatter is larger for fainter limiting magnitudes, i.e. higher source redshifts.
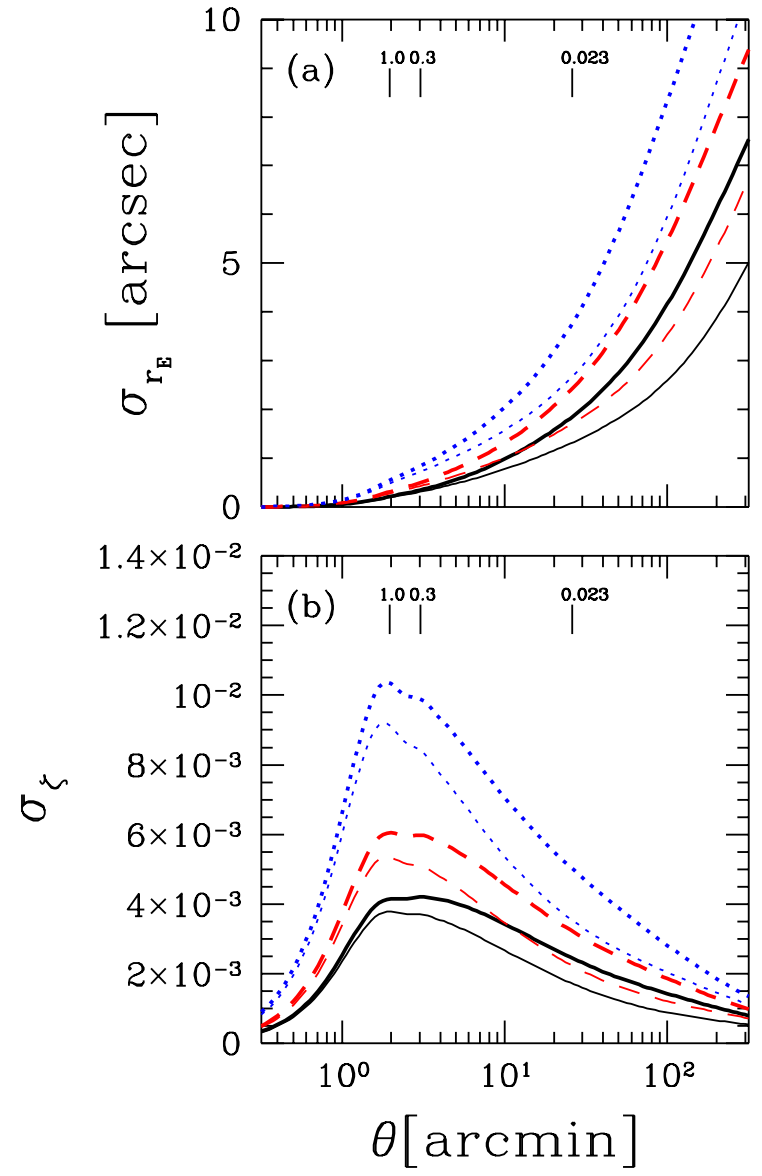

Fig. 3. a) Scatter in the estimate of the Einstein radius $r_{\mathrm{E}}$ due to large scale structure for the SCDM model (thick lines) and the OCDM model (thin lines). We use three different redshift distributions for each model. These are based on photometric redshifts inferred from both HDFs taking different limiting magnitudes. The solid line corresponds to $20<R<22$, the dashed line to $20<R<24$, and the dotted line to $20<R<26$. We have indicated a radius of $1 h_{50}^{-1} \mathrm{Mpc}$ for clusters at various redshifts. Panel $\mathbf{b}$ ) shows the results for the $\zeta$-statistic as a function of aperture size

Figure $3 \mathrm{~b}$ shows the scatter for the $\zeta$-statistic. Because both $\left\langle\zeta_{\text {LSS }}^{2}\right\rangle^{1 / 2}$ and $\bar{\kappa}(<\theta)$ decrease with increasing aperture size the interpretation of Fig. $3 \mathrm{~b}$ is not as straightforward as is the case for Fig. 3a. The effect of aperture size on the accuracy of weak lensing mass estimates is discussed in detail in Sect. 4.3.

Comparison of the results for the SCDM and OCDM cosmologies indicates that the differences are fairly small. We found that the $\Lambda$ CDM cosmology gives similar estimates. Although we consider these different cosmologies throughout the paper (and plot the results for the OCDM and SCDM models) we note that the results hardly depend on the assumed cosmology.

\section{Results}

We examine the uncertainty in the weak lensing mass estimate caused by uncorrelated large scale structure. 

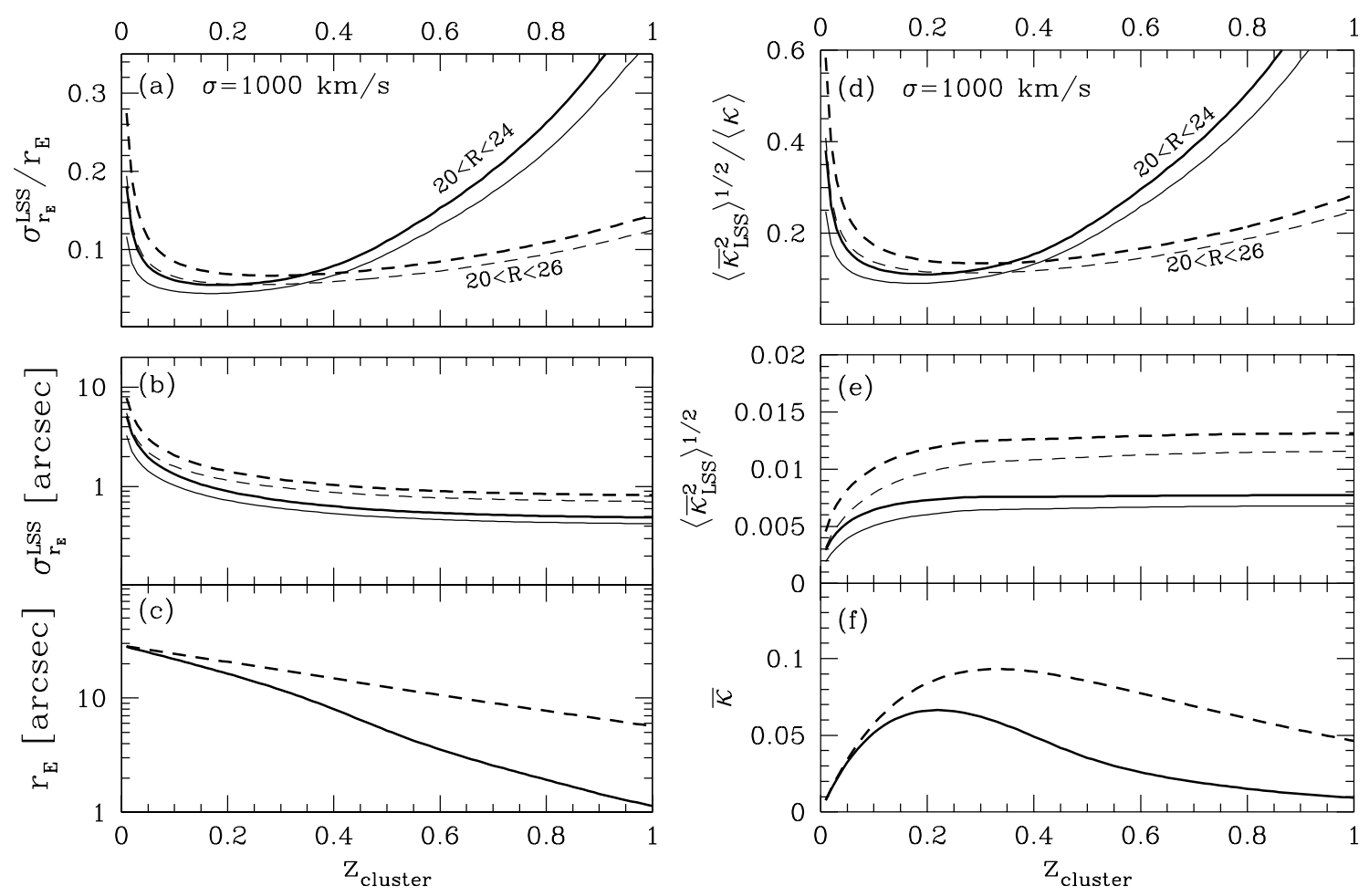

Fig. 4. a) Relative importance of lensing by distant large scale structure on the measurement of the Einstein radius $r_{\mathrm{E}}$ as a function of cluster redshift, when the shear is measured in an annulus from 0.225 to $1.5 h_{50}^{-1} \mathrm{Mpc}$. b) The scatter in the measurement of $r_{\mathrm{E}}$ caused by large scale structure. c) The observed Einstein radius as a function of cluster redshift. d) Same as panel a), but now when the $\zeta$-statistic is used to estimate $\bar{\kappa}\left(<1 h_{50}^{-1} \mathrm{Mpc}\right)$. We have assumed that the lensing signal is measured out to $1.5 h_{50}^{-1} \mathrm{Mpc}$ from the cluster centre, and that the average surface density $\bar{\kappa}\left(1<r<1.5 h_{50}^{-1} \mathrm{Mpc}\right)$ is determined from a SIS model fit to the tangential shear. e) Scatter in $\bar{\kappa}$ because of large scale structure. f) $\bar{\kappa}\left(<1 h_{50}^{-1} \mathrm{Mpc}\right)$ as a function of cluster redshift. The thick lines correspond to the SCDM model, and the thin lines to the open model. The values for either $r_{\mathrm{E}}$ and $\bar{\kappa}$ turn out to be almost identical for these two cosmologies

The results are derived for a cluster with a SIS mass profile, and a velocity dispersion of $\sigma=1000 \mathrm{~km} \mathrm{~s}^{-1}$, typical for rich clusters that have been studied in the past. We study the dependence on cluster redshift, where we discuss the results for low redshift clusters in more detail. We also examine how the accuracy of the mass measurements depends on aperture size.

\subsection{Dependence on cluster redshift}

First we examine how the mass estimates for clusters at different redshifts are affected when the lensing signal is measured out to a radius of $1.5 h_{50}^{-1} \mathrm{Mpc}$.

For the SIS model we use the measurements in an annulus from 0.225 to $1.5 h_{50}^{-1} \mathrm{Mpc}\left(R=1.5 h_{50}^{-1} \mathrm{Mpc}\right.$, $\alpha=0.15)$. In the case of the $\zeta$-statistic we use an inner radius of $\theta_{1}=1 h_{50}^{-1} \mathrm{Mpc}$ and an outer radius of $\theta_{2}=1.5 h_{50}^{-1} \operatorname{Mpc}\left(\alpha=\frac{2}{3}\right)$. The value of the $\zeta$-statistic only provides a lower limit to the cluster mass, and one way to circumvent this is to estimate the average surface density in the annulus around the aperture, for instance by using the results from a model fit. However, this model fit is also affected by large scale structure. In general the region where the model is fitted to the data largely coincides with the annulus used to compute the value of the $\zeta$-statistic. Thus if the value of the $\zeta$-statistic is increased by additional structures, the mass from the model will also be higher. Although the uncertainty in the model parameters and the $\zeta$-statistic are not completely correlated, we will assume they are. This gives a fair upper limit on the uncertainty in the estimate of $\bar{\kappa}$

$\sigma_{\bar{\kappa}}^{\mathrm{LSS}}=\sigma_{\zeta}^{\mathrm{LSS}}+\frac{\sigma_{r_{\mathrm{E}}}^{\mathrm{LSS}}}{(1+\alpha) \theta_{2}}$.

The results are presented in Fig. 4. Panels c) and f) show respectively the value of the Einstein radius and $\bar{\kappa}$ as a function of cluster redshift. We show the results for the SCDM (thick lines) and the OCDM (thin lines) cosmologies. The solid lines are for a limiting magnitude of $R=24$ and the dashed lines for $R=26$. Panels b) and e) show the scatter in the aperture mass statistics introduced by the large scale structure. Finally the ratios of the scatter and the lensing signal are presented in Figs. 4a and d.

For clusters at low redshifts $(z<0.2)$ the relative importance of large scale structure for the $\Lambda \mathrm{CDM}$ model is similar to that of the SCDM model. For clusters at high 


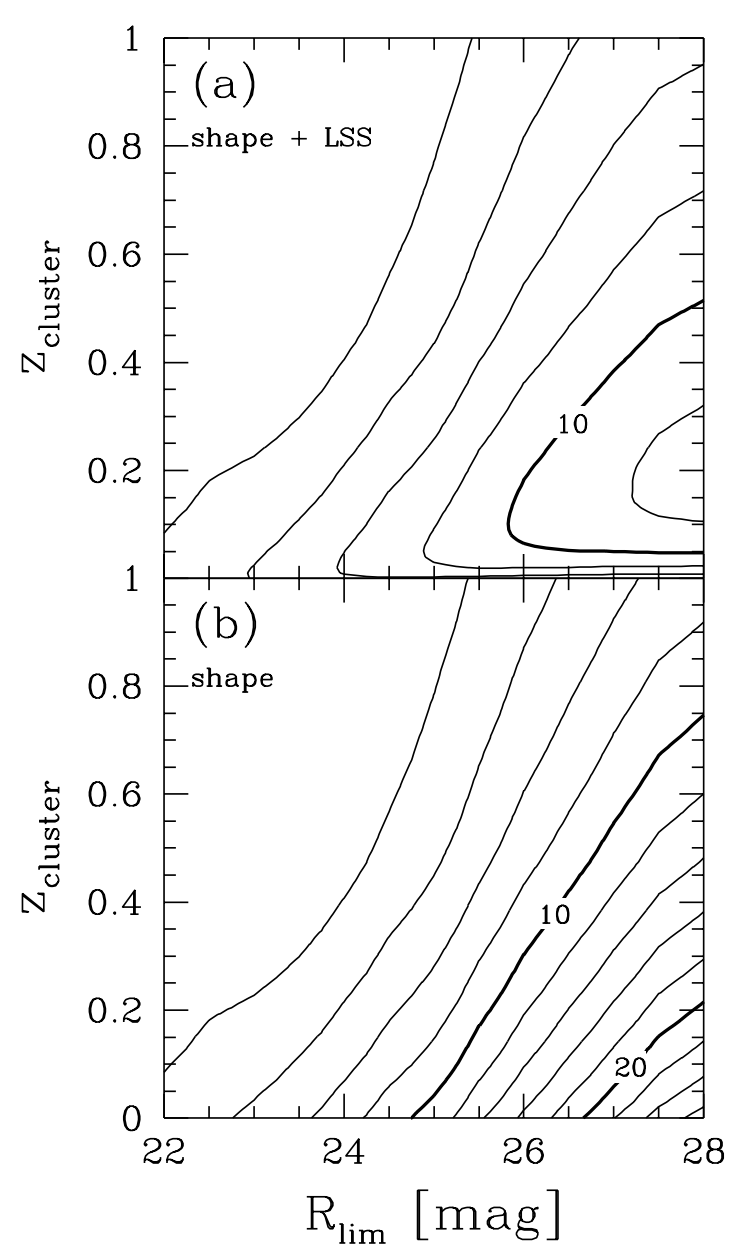

Fig. 5. a) Contour plot of the signal-to-noise ratio for a SIS model fit to the tangential shear of a cluster of $\sigma=1000 \mathrm{~km} \mathrm{~s}^{-1}$ as a function of limiting magnitude $R_{\text {lim }}$ and cluster redshift $z_{c}$. The noise includes the contribution of the intrinsic shapes of the sources and the contribution by large large scale structure for the OCDM cosmology. b) Contour plot of the signal-tonoise ratio when the noise caused by large scale structure is ignored. The interval between adjacent contours is 2

redshift, or deep observations, the results for the $\Lambda \mathrm{CDM}$ are close to the OCDM results.

Comparison of Figs. $4 \mathrm{a}$ and $\mathrm{d}$ shows that the relative importance of the distant large scale structure is smaller in the case of the SIS model fit. For this mass estimator the uncertainty in the cluster mass estimate is about $6 \%$ for rich clusters at intermediate redshifts. The uncertainty in the mass is large for high redshift clusters when the limiting magnitude of the sources is rather bright (solid lines). However, in practice, to detect a significant lensing signal of a high redshift cluster deep observations are needed to ensure a sufficiently high number density of sources. As a result the importance of large scale structure is minimized (dashed lines). In the case of nearby clusters, the presence of structure along the line of sight results in a relatively large uncertainty in the cluster mass (see Sect. 4.2).
The uncertainty introduced by large scale structure is much larger when the $\zeta$-statistic is used. This is not surprising as one only uses the shear measurements at relatively large radii, where the cluster mass surface density has become fairly low. In addition to this annulus, the SIS model fit uses data at much smaller radii, where the cluster signal is much higher.

The noise in weak lensing analyses is generally dominated by the intrinsic shapes of the source galaxies, and not by the noise in the large scale structure. Both sources of error depend differently on the limiting magnitude of the background galaxies, and as a result the total noise is a rather complex function of cluster redshift and limiting magnitude, as is demonstrated in Fig. 5a for the OCDM cosmology.

Figure $5 \mathrm{~b}$ shows the the signal-to-noise ratio for a SIS model fit to the tangential shear of a cluster of $\sigma=1000 \mathrm{kms}^{-1}$, when the contribution of large scale structure is ignored. It should be noted here that we assume that the shapes of the sources have been measured accurately. In reality the shapes of very faint galaxies are difficult to determine. From Fig. 5b one would expect to be able to measure accurate masses for low redshift clusters. However, the results change significantly when we include the contribution from large scale structure as is shown in Fig. 5a. The change in signal-to-noise ratio is small for high redshift clusters, where the shapes are still the major contributor to the noise. The true signal-to-noise ratio is lowered significantly in the case of deep observation of clusters at intermediate redshifts, but the largest difference is seen for clusters at low redshifts.

The signal-to-noise ratios for the $\Lambda \mathrm{CDM}$ model are intermediate to those found for the OCDM and SCDM models, resembling the OCDM better for high redshift clusters. However, at high redshifts the differences between the various models become less important (they are fairly small anyway), because the noise is dominated by the intrinsic shapes of the sources.

\subsection{Low redshift clusters}

Figure 5a demonstrates that large scale structure introduces a large uncertainty in the mass measurement of low redshift clusters. To date not many low redshift clusters have been studied through weak lensing. This is because the amplitude of the lensing signal peaks at intermediate redshifts, and the signal is extremely small for nearby clusters. Furthermore their angular sizes were too large for the CCD cameras. Panoramic CCD cameras have become available recently, and a number of low redshift clusters have been imaged for weak lensing purposes. For instance Joffre et al. (2000) have analysed the mass distribution of Abell 3667 ( $z=0.055)$.

Although the lensing signal of low redshift clusters is small, there are several reasons to study these systems. First of all, they can, or have been studied extensively by other techniques. Also, the uncertainty in the redshift 


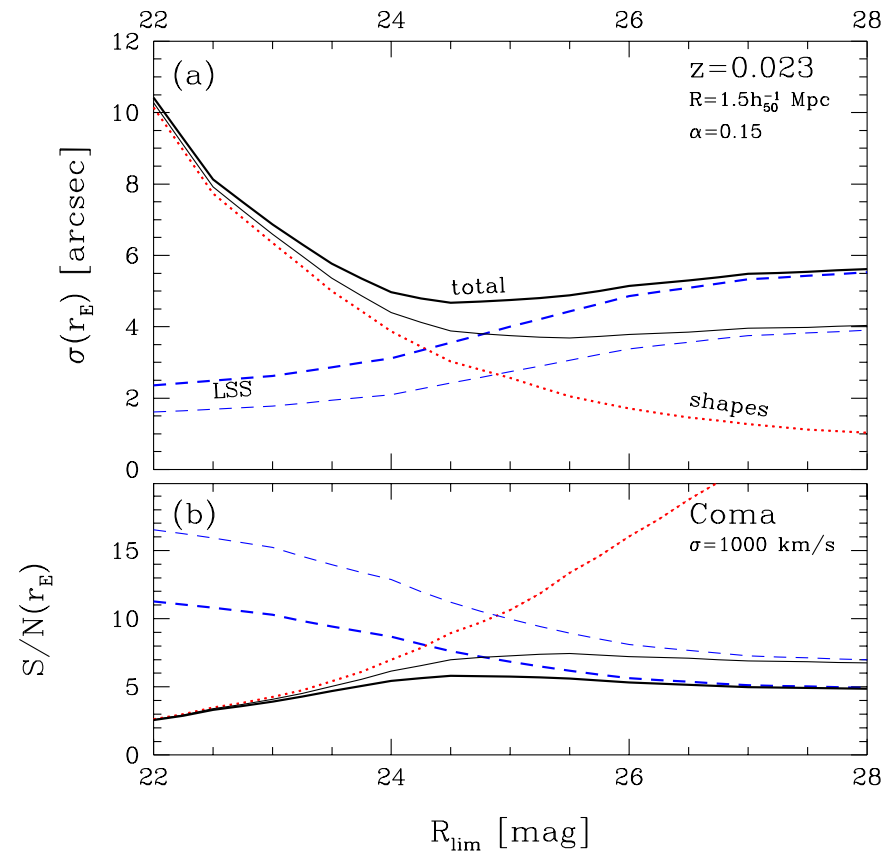

Fig. 6. a) Sources of noise in the measurement of the Einstein radius $r_{\mathrm{E}}$ from a SIS model fit. The figure shows the noise caused by the large scale structure (dashed lines) and the intrinsic shapes of the sources (dotted lines) as a function of limiting magnitude $R_{\mathrm{lim}}$ of the sources. The thick lines correspond to the SCDM model and the thin lines to the OCDM cosmology. The results for the $\Lambda \mathrm{CDM}$ model are similar to the SCDM model. b) Expected signal-to-noise ratio as a function of limiting magnitude of the sources for the Coma cluster. For limiting magnitudes $R>24$ the signal-to-noise ratio of the measurement of the Einstein radius no longer increases, because of the noise from the large scale structure. The results for the $\Lambda \mathrm{CDM}$ model lie between the drawn curves

distribution of the sources is almost non-existent, and one can use relatively large background galaxies.

In this section we examine how the weak lensing mass measurement of the Coma cluster $(z=0.023)$ is affected by structures at higher redshifts. A weak lensing analysis of the most nearby rich cluster of galaxies is challenging, but could provide important insights in the cluster dynamics. We assume a velocity dispersion of $1000 \mathrm{~km} \mathrm{~s}^{-1}$ for the cluster (e.g., Colles \& Dunn 1996).

In Fig. 6a we plot the sources of noise in the measurement of the Einstein radius as a function of limiting magnitude of the sources. The figure shows the contribution from the large scale structure (dashed lines) and the intrinsic shapes of the sources (dotted lines). For the intrinsic shapes of the sources we have assumed $\left\langle\gamma_{T}^{2}\right\rangle^{1 / 2}=0.25$, similar to what is observed in deep HST images (e.g., Hoekstra et al. 2000) Reaching the depth of the HDFs one would expect to obtain a signal-to-noise ratio of more than 20. However, Fig. 6a demonstrates that the total uncertainty in the measurement of the Einstein radius reaches a minimum value for a limiting $R=24$.

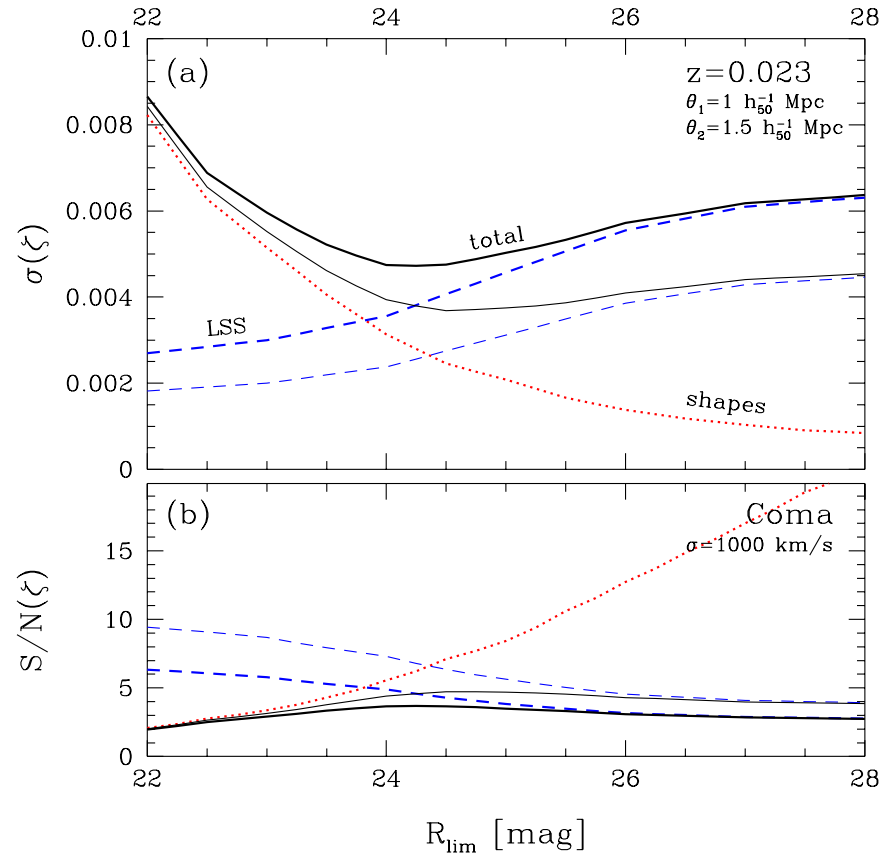

Fig. 7. As Fig. 6 but now for the $\zeta$-statistic. The noise introduced by the large scale structure results in a constant signalto-noise ratio for limiting magnitudes $R>25$. Note that the achieved signal-to-noise ratio for the $\zeta$-statistic is lower in comparison to the SIS model fit

Figure $6 \mathrm{~b}$ shows the expected signal-to-noise ratio as a function of limiting magnitude, and it demonstrates that observations with $R_{\text {lim }}>24.5$ no longer improve the signal-to-noise ratio of the mass measurement. The maximum signal-to-noise ratio that can be reached by fitting a SIS model to the observed tangential shear around the Coma cluster is $\sim 5$ for the SCDM model, and $\sim 7$ for the OCDM model. The results for the $\Lambda$ CDM model lie between the curves for the other two models.

In Fig. 7 we show how the accuracy of the mass measurement is affected by large scale structure when the $\zeta$ statistic is used. Comparison with Fig. 6 shows that the SIS model fit yields a better signal-to-noise ratio.

\subsection{Large apertures}

The intrinsic shapes of the sources result in a statistical uncertainty in the measurement of the Einstein radius. If the SIS model is fitted to the measurements in an annulus from $R_{0}$ out to $R$, the error in $r_{\mathrm{E}}$ is given by

$\sigma_{r_{\mathrm{E}}}=\sqrt{\frac{2}{\pi \bar{n} \ln \left(R / R_{0}\right)}} \sigma_{\mathrm{gal}}$,

where $\bar{n}$ is the number density of sources, and $\sigma_{\text {gal }}$ is the uncertainty in the measurement of the tangential distortion for a single galaxy. 


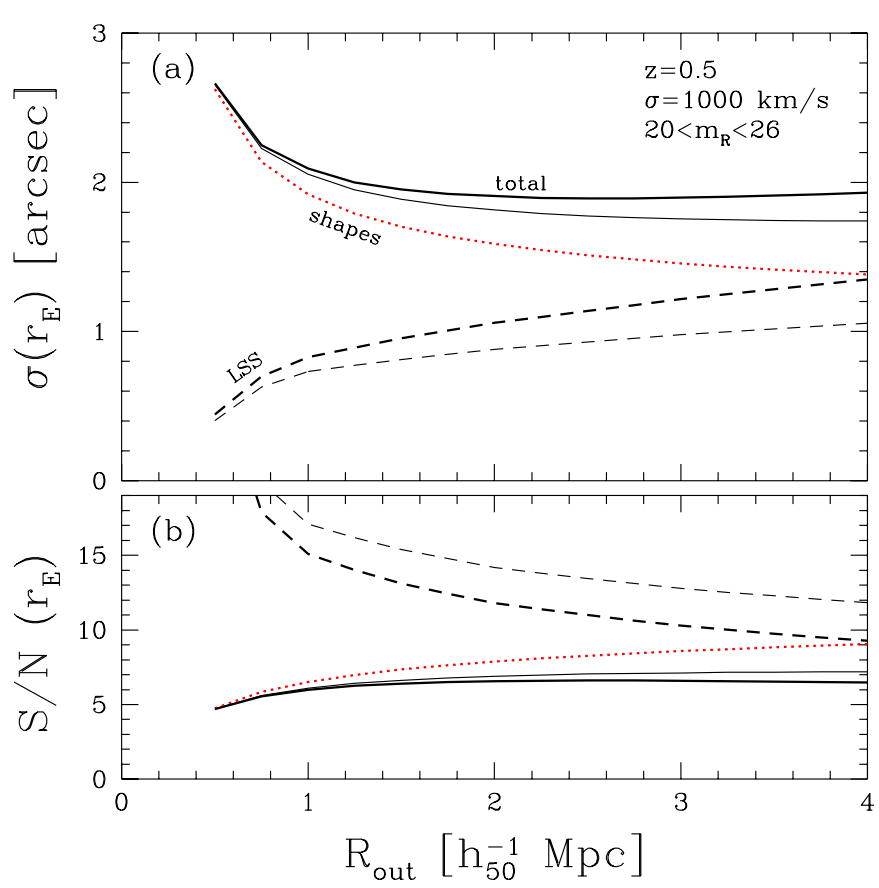

Fig. 8. a) Error on the measurement of the Einstein radius $r_{\mathrm{E}}$ as a function of aperture size, because of the large scale structure (dashed lines) and the instrinsic shapes of the sources (dotted lines). The SIS model is fitted to the tangential shear in an annulus ranging from $R_{0}=0.225 h_{50}^{-1} \mathrm{Mpc}$ out to $R_{\text {out }}$. The thick lines give the results for the SCDM model, whereas the thin lines correspond to the OCDM model. b) The expected signal-to-noise ratio as a function of aperture size. The curves for the $\Lambda$ CDM model are comparable to the results for the OCDM model. The signal-to-noise ratio does not improve for aperture sizes $R_{\text {out }}>1 h_{50}^{-1} \mathrm{Mpc}$

From Eq. (26) we find that this contribution to the error budget decreases slowly for increasing aperture sizes. However, Fig. 3a shows that the noise contribution from large scale structure increases rather rapidly with aperture size.

Figure $8 \mathrm{a}$ shows how the contributions of the intrinsic shapes of the sources, and the large scale structure depend on the aperture size. The results are for a cluster at a redshift $z=0.5$, and the lensing signal has been measured from $R_{0}=0.225 h_{50}^{-1} \mathrm{Mpc}$ out to a radius $R_{\text {out }}$. The uncertainty in $r_{\mathrm{E}}$ caused by the intrinsic shapes of the sources decreases slowly with aperture size (dotted curve), whereas the contribution by the distant large scale structure increases (dashed line). Figure $8 \mathrm{~b}$ shows the resulting signal-to-noise ratio. The signal-to-noise ratio is virtually constant for outer radii larger than $\sim 1 h_{50}^{-1} \mathrm{Mpc}$. Measuring the lensing signal in larger apertures does not improve the accuracy of the cluster mass. One has also to keep in mind that we have assumed that the mass profile is well described by a SIS model, which might not be true in the outer parts of clusters.

For clusters at higher redshifts the curves look similar, but the maximum signal-to-noise ratio is reached at some- what larger (physical) aperture sizes. The noise caused by the large scale structure dominates the error for low redshift clusters, and for these systems, the signal-to-noise ratio decreases slowly with increasing aperture size.

So far we have used an inner radius for the SIS model fit of $R_{0}=0.225 h_{50}^{-1} \mathrm{Mpc}$, but one cannot always fit the model at such small distances from the centre. For instance in the case of the $z=0.83$ cluster MS 1054-03 Hoekstra et al. (2000) showed that the mass distribution in the centre is rather complex and the substructure lowers the azimuthally averaged tangential shear. Consequently they fit the SIS model from $\sim 0.7$ out to $\sim 2 h_{50}^{-1} \mathrm{Mpc}$, which corresponds to $\alpha=0.35$.

We also have examined how the accuracy of the mass estimate changes when the SIS model is fitted to the data within a given aperture, while the inner radius is increased. In this situation both the noise caused by the shapes of the sources and the contribution by the large scale structure increase. Thus small values for the inner radius improve the signal-to-noise of the mass measurement, but one should keep in mind that substructure in the cluster centre complicates a safe choice for the inner radius.

For the $\zeta$-statistic the noise contribution caused by the shapes of the galaxies is given by

$$
\sigma_{\zeta}=\sqrt{\frac{1}{\pi\left(1-\alpha^{2}\right) \theta_{1}^{2} \bar{n}}} \sigma_{\text {gal }}
$$

However, we want to estimate $\bar{\kappa}\left(<1 h_{50}^{-1} \mathrm{Mpc}\right)$, and thus we have to add the average surface density in the annulus from 1 to $R_{\text {out }} h_{50}^{-1}$ Mpc. As before we estimate $\bar{\kappa}(1<$ $\left.r<R_{\text {out }}\right)$ from a SIS model fit to the tangential shear. The errors in the $\zeta$-statistic and the Einstein radius from the model fit are combined according to Eq. (25), because the errors are correlated. We note that the size of the correction depends on the assumed mass profile, but for increasing values of $R_{\text {out }}$ the correction becomes smaller (i.e., $\bar{\kappa}\left(1<r<R_{\text {out }}\right)$ becomes smaller).

The resulting noise estimates are presented in Fig. 9a. The corresponding signal-to-noise ratio of $\bar{\kappa}\left(<1 h_{50}^{-1} \mathrm{Mpc}\right)$ for a cluster at a redshift $z=0.5$ is presented in Fig. $9 \mathrm{~b}$. It shows that using a larger $R_{\text {out }}$ improves the accuracy in the measurement of $\bar{\kappa}\left(<1 h_{50}^{-1} \mathrm{Mpc}\right)$.

A similar calculation shows that the contribution of the large scale structure becomes more important when one tries to measure the average surface density in larger apertures. For instance the uncertainty in $\bar{\kappa}\left(<2 h_{50}^{-1} \mathrm{Mpc}\right)$ almost doubles compared to $\bar{\kappa}\left(<1 h_{50}^{-1} \mathrm{Mpc}\right)$.

Attempts to measure the average cluster mass profile out to large projected distances from the cluster centre will also be affected by large scale structure, given the fact that it is already difficult to measure the total mass. Unfortunately it will be difficult to quantify the effect of distant large scale structure on such measurements. 


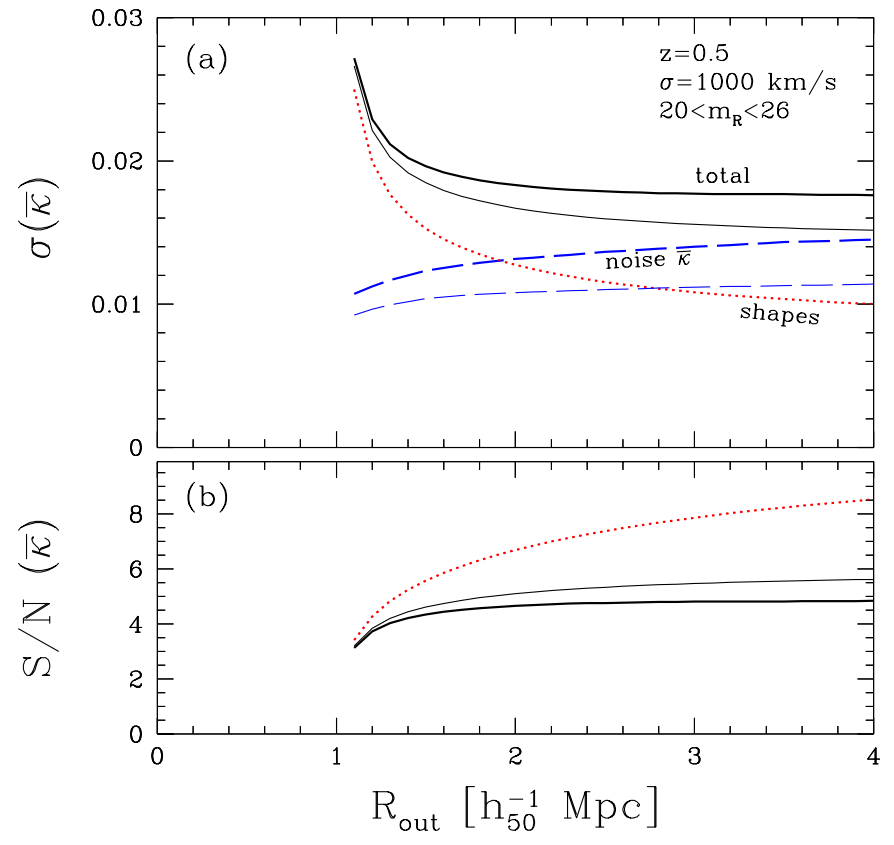

Fig. 9. As Fig. 8, but now we show the measurement error on $\bar{\kappa}\left(<1 h_{50}^{-1} \mathrm{Mpc}\right)$ as a function of $R_{\text {out }}$ when the $\zeta$-statistic is used to estimate the mass. The average surface density in the annulus from 1 to $R_{\text {out }} h_{50}^{-1} \mathrm{Mpc}$ is estimated from a SIS model fit to the observations. The thick lines give the results for the SCDM model, whereas the thin lines correspond to the OCDM model. b) The expected signal-to-noise ratio of $\bar{\kappa}\left(<1 h_{50}^{-1} \mathrm{Mpc}\right)$ as a function of aperture size. The signal-tonoise ratio improves with increasing $R_{\text {out }}$. If the cluster mass profile is well described by a SIS model, the model fit gives the better results

\section{Conclusions}

The observed weak lensing signal is sensitive to all matter along the line of sight, and as a result this additional matter acts as a source of uncertainty in mass estimates of clusters of galaxies. In this paper we have investigated the effect of distant large scale structure, uncorrelated with the cluster mass distribution. It can be treated as an additional source of noise.

Given a cosmological model it is possible to compute the statistical uncertainty in the weak lensing mass measurement caused by the distant large scale structure, and quantify its contribution to the total error budget. We have considered two methods to estimate the mass from a weak lensing analysis. One way is to fit a SIS model to the observed lensing signal. The other method uses the $\zeta$ statistic (e.g., Fahlman 1994) to estimate the mass. Both methods give similar results.

We find that the importance of matter along the line sight is fairly small for rich clusters $\left(\sigma=1000 \mathrm{~km} \mathrm{~s}^{-1}\right)$ at intermediate redshifts, provided that the bulk of the sources are at high redshift compared to the cluster. If the lensing signal is measured out to $1.5 h_{50}^{-1} \mathrm{Mpc}$ from the cluster centre, the typical $1 \sigma$ relative uncertainty in the mass cause by the large scale structure is about $6 \%$.
In certain situations the effect of large scale structure is more important. For nearby clusters, such as the Coma cluster, background structures introduce a considerable uncertainty in the mass. Considering only the noise caused by the shapes of the sources used in the weak lensing analysis one expects to achieve very accurate weak lensing mass measurements of nearby clusters, but we find that the large scale structure limits the maximum achievable signal-to-noise ratio to $\sim 7$, irrespective of the depth of the observations.

We also have examined how the accuracy of the weak lensing mass estimate changes when the lensing signal is measured out to large projected distances from the cluster centre. In the case of the SIS model fit we find that the signal-to-noise ratio does not increase significantly when one includes measurements at radii larger than $\sim 1 h_{50}^{-1} \mathrm{Mpc}$. When the $\zeta$-statistic is used to estimate the mass within a $1 h_{50}^{-1} \mathrm{Mpc}$ radius aperture the signal-to-noise ratio does increase with $R_{\text {out }}$. If the cluster mass profile well described by a SIS model, the model fit results in a better signal-to-noise ratio.

The effect of large scale structure is important in the outskirts of clusters, where the cluster signal itself is low. In this paper we have quantified the uncertainty in the measurement of the cluster mass, but distant large scale structure will also introduce noise in studies that try to constrain the mass profiles of clusters at large projected distances. Furthermore studies of the 3-D structure of clusters by combining weak lensing, X-ray, and S-Z data (e.g., Zaroubi et al. 1998) will be affected by uncorrelated large scale structure.

Photometric redshifts of the sources can be used to decrease the effect of large scale structure, either by modeling the contribution of large scale structure to the lensing signal, or by selecting sources in an optimal redshift range. This can be only done at the expense of a large amount of additional data. Because the effect of large scale structure can be treated as an additional (statistical) source of noise. Thus an alternative procedure is to average the results of an ensemble of clusters, thus decreasing the noise on the (average) cluster mass estimate.

Acknowledgements. We thank the referee for several useful comments which improved the paper. It is a pleasure to thank Marijn Franx and Konrad Kuijken for useful discussions and comments to improve the paper. Also the comments from Ludo van Waerbeke are acknowledged. Peter Schneider made available his algorithms, which were very helpful.

\section{References}

Bacon, D., Refregier, A., \& Ellis, R. S. 2000, MNRAS, 318, 625

Bartelmann, M., \& Schneider, P. 1999, submitted to Phys. Rep. [astro-ph/9912508]

Bernardeau, F., van Waerbeke, L., \& Mellier, Y. 1997, A\&A, 322,1

Blandford, R. D., Saust, A. B., Brainerd, T. G., \& Villumsen, J. V. 1991, MNRAS, 251, 600

Cen, R. 1997, ApJ, 485, 39 
Chen, H.-W., Fernández-Soto, A., Lanzetta, K. M., et al., preprint [astro-ph/9812339],

http://www.ess.sunysb.edu/astro/hdfs/home.html

Colless, M., \& Dunn, A. M. 1996, ApJ, 458, 435

Fahlman, G., Kaiser, N., Squires, G., \& Woods, D. 1994, ApJ, 437, 56

Fernández-Soto, A., Lanzetta, K. M., \& Yahil, A. 1999, ApJ, 513, 34

Gorenstein, M. V., Shapiro, I. I., \& Falco, E. E. 1988, ApJ, 327,693

Hamilton, A. J. S., Kumar, P., Lu, E., \& Matthews, A. 1991, ApJ, 374, L1

Hoekstra, H., Franx, M., Kuijken, K., \& Squires, G. 1998, ApJ, 504,636

Hoekstra, H., Franx, M., \& Kuijken, K. 2000, ApJ, 532, 88

Jain, B., \& Seljak, U. 1997, ApJ, 484, 560

Joffre, M., Fischer, P., Frieman, J., et al. 2000, ApJ, 534, L131
Kaiser, N. 1992, ApJ, 388, 272

Kaiser, N., Wilson, G., \& Luppino, G. A. 2000, ApJL, submitted [astro-ph/0003338]

Mellier, Y. 1999, ARA\&A, 37, 127

Metzler, C. A., White, M., Norman, M., \& Loken, C. 1999, ApJ, 520, L9

Peacock, J. A., \& Dodds, S. J. 1996, MNRAS, 780, L19

Schneider, P. 1996, MNRAS, 283, 837 (S96)

Schneider, P., van Waerbeke, L., Jain, B., \& Kruse, G. 1998, MNRAS, 296, 873 (SWJK)

Tyson, J. A., Wenk, R. A., \& Valdes, F. 1990, ApJ, 349, 1

van Waerbeke, L., Mellier, Y., Erben, T., et al. 2000, A\&A, 358,30

Wittman, D. M., Tyson, J. A., Kirkman, D., Dell'Antonio, I., \& Bernstein, G. 2000, Nature, 405, 143

Zaroubi, S., Squires, G., Hoffman, Y., \& Silk, J. 1998, ApJ, 500,87 